\title{
Hardware Implementation of Hybrid Renewable Energy Source for Integrated Fed Micro-Grid
}

\author{
Dr. Balachandra Pattanaik ${ }^{1}$, Dr.K.Sakthivel ${ }^{2}$, Dr.S.Priya ${ }^{3}$ \\ balapk1971@gmail.com ${ }^{1}$, sakthivelk.eee@bharathuniv.ac.in ${ }^{2}$, \\ priya.s@ametuniv.ac.in ${ }^{3}$
}

Bule Hora University, Ethiopia,Africa ${ }^{1}$, Bharath Institute of Higher Education and Research, Chennai, Tamil Nadu, India ${ }^{2}$, AMET Deemed to be University, Chennai, Tamil Nadu, India ${ }^{3}$

\begin{abstract}
Because of the rapid development progress of Power natural philosophy strategies, applications with electrical phenomenon (PV) power and wind generation are swollen basically the employment of sun supercharged And wind energies severally wouldn't offer an immersed yield voltage that the solar power and Wind energy square measure consolidated can improve the characteristics of every alternative. To diminish the ability demand on the normal power age space, we have a tendency to propose this method. Various ways square measure by and by for age of intensity utilizing SolarWind Hybrid System with most electrical outlet pursuit (MPPT). Steady voltage strategy is used for much extreme power exchange. This strategy has to be compelled to have some key highlights to create the potency. Above all this article proposes a completely unique technique for implementing MPPT controller in our hybrid renewable energy generation system known as FLC rule for higher potency than alternative strategies. The higher than explained system is intended and modeled for output results were obtained.
\end{abstract}

Keywords: Fuzzy Logic( FL) algorithm, Maximum Power Point Tracking (MPPT).

\section{Introduction}

A making system with a solitary power supply non-traditional energy [6] doesn't provide power needed due to warming, new energy sources ought to be utilized for instance, sun destined and wind energy property power supply is planning to be additional vital. Sun destined and Wind energy is while not contamination and endless. All districts of the globe have inexhaustible assets of some kind. Hybrid system could be a high performance compared to unconventional supply. As brooding about these 2 energy assets, the yield is not certain due to ecological or day and night circumstances [9-12]. That's the yield of Solar-Wind power is not certain one could offer additional power and different could offer less power or no power. Thus it needed to balance out yield voltage from this method. These 2 systems associated parallel to every different, that within the event that one supply is not accessible, at that time the opposite one will modify the system [15]. During this manner, these 2 systems will work on an individual basis and at constant time moreover. During this paper a replacement technique mistreatment FLC algorithmic rule for the planned MPPT for the hybrid grid is enforced for providing higher potency and dependableness.

\section{Proposed Hybrid System}

During this paper a noval methodology for MPPT controller is enforced for the aim of higher potency from each wind and alternative energy system the tactic used here is formal logic Controller (FLC) formula. The turbine used here is magnet Synchronous Generator (PMSG) with parallel affiliation of electrical phenomenon power generation with battery bank for providing higher dependableness. 
The electrical converter used here is electrical phenomenon supply electrical converter that is controlled by PI controller the facility from our hybrid grid is reborn from DC to AC through ZSI (Z-Source Inverter) and also the extracted power is in addition to the grid and may be provided to the specified load if required.

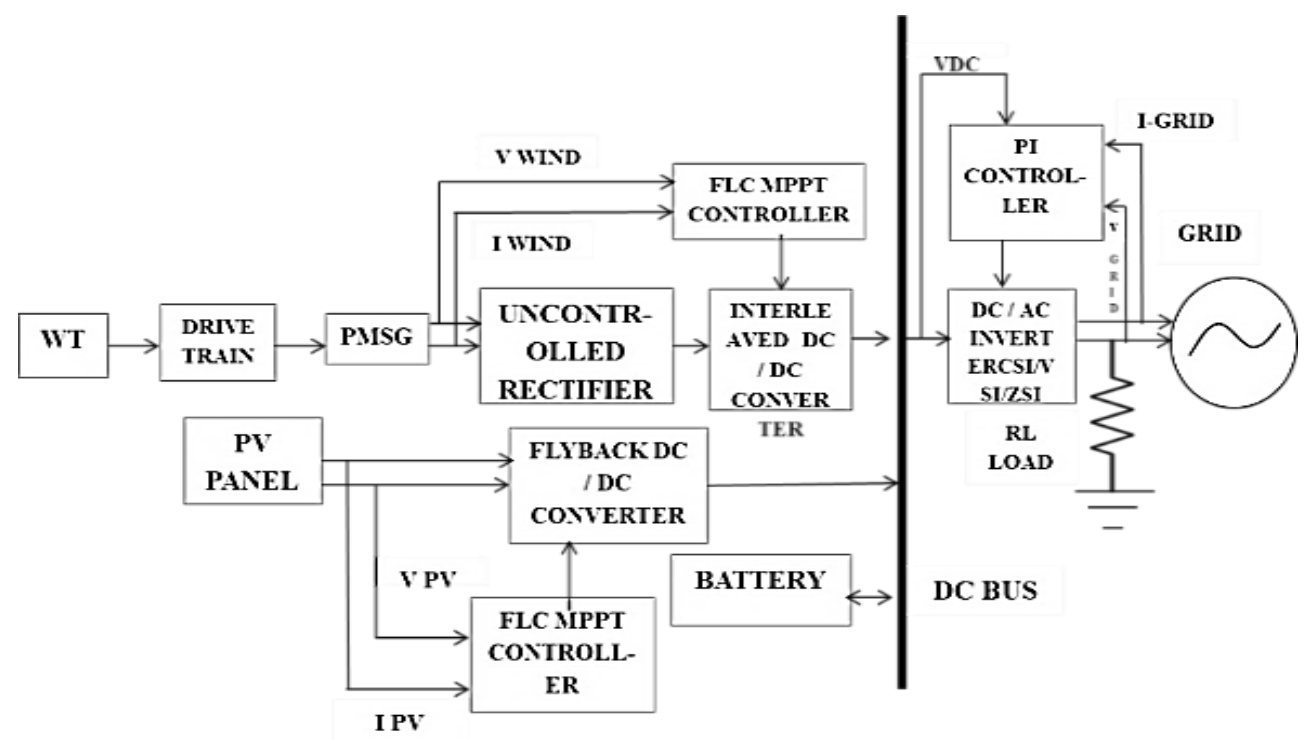

Fig.1 Block illustration of planned Hybrid Renewable Energy Fed micro-grid

\subsection{Microgrid}

Microgrid represents a completely new approach to desegregation Distributed Energy Resources (DER), particularly tiny generators, into utility distribution systems. Ancient approaches for desegregation DER specialize in the impacts on grid performance of 1, two, or a comparatively tiny variety of on an individual basis interconnected micro generators. An important feature of the Microgrid is its presentation to the encompassing distribution grid as one manageable system. Key to the present characteristic is reliance on the flexibleness of advanced power natural philosophy that controls the interface between micro sources and therefore the close AC system.

The central advantage of a Microgrid is that it will be considered a controlled part inside the facility system which will be operated as one dispatchable load, which is able to respond in seconds to distribution system desires. Customers additionally get pleasure from a Microgrid that's designed to fulfill their native desires, e.g., for uninterruptible power supply/enhanced native dependability, reduced feeder losses, supported native voltages/correction of voltage sag, and exaggerated potency through use of waste heat.

\subsection{Fuzzy Logic Controller}

Fuzzy Logic may be a specific space of concentration within the study of computer science and relies on the worth of that data that is neither undoubtedly true nor false. No inheritable information is a robust weapon to combat the unsought effects of the system response. In most applications there are some points that are the common space. Data that lies among the common space needs to be studied, stored, and accustomed quantify and to classify the information. This enables for good manipulation of the information structure so as to form illation to an answer. Data that falls in this common space will be stratified, aged, and "best guess" created when analysis of this "gray" data. 


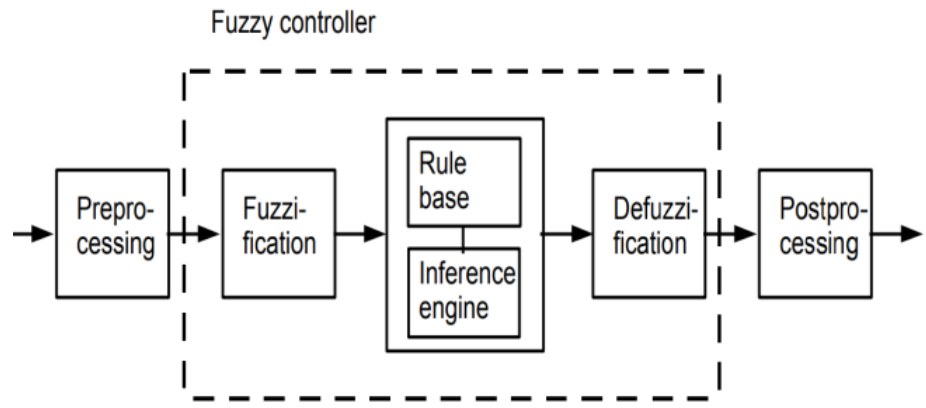

Fig.2 Fuzzy controller Working Method

\subsection{Interleaved boost converter}

An Interleaved boost converter ads further benefits like reduced ripple currents in each the input and output circuits. Higher potency is complete by ripping the output current into 2 ways, well reducing I2R losses and electrical device AC losses. Figure one shows the fundamental interleaved boost topology. When s1 activates, current ramps up in L1 and slope reckoning on the input voltage, storing energy in L1. D1 is off throughout this point since the output voltage is bigger than the input voltage. Once s1 turns off, D1 conducts delivering a part of its keep energy to the load and therefore the output condenser. Current in L1 ramps down with a slope keen to the distinction between input and output voltage. One half a switching later, s2 conjointly activates finishing identical cycle of events. Since each power channels are combined at the output condenser, the effective ripple frequency is double that of a traditional single channel boost regulator.

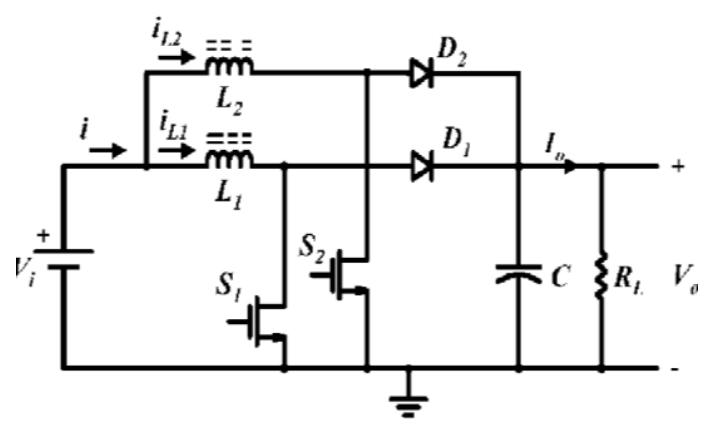

Fig.3 Interleaved boost converter

The interleaved boost converter will increase the voltage output to the twice the amount from the input voltage. This unique quality makes this converter more efficient but it has its own draw backs as it has two semiconductor switches will increase semiconductor loss.

\section{Designing and implementation of proposed method for maximum power extraction}

This proposed maximum power extraction system consists of various elements to tune the system for better performance, those techniques which were explained above is implemented in hardware. The total proposed system hardware model is shown in the figure 4 . The power 
generated from solar and wind power is fed into interleaved boost converter and it is further sent to inverter circuit where the proposed PWM technique is used to give gate pulse to the semiconductor devices present in the circuit. The method used for improving the dc voltage from solar is MPPT controller. The solar MPPT model is modeled with PIC microcontroller as shown in the figure 4.

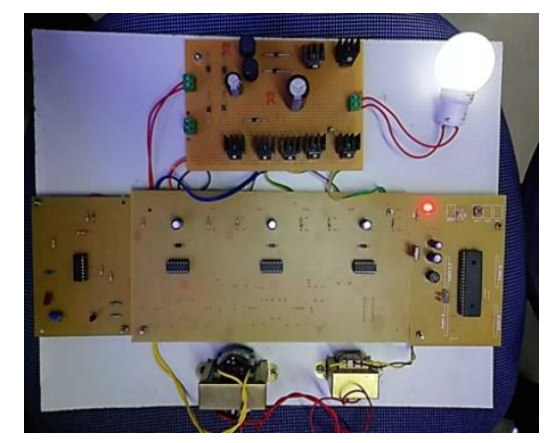

Fig.4. Simulation model for proposed hybrid wind solar generation system The wind power system consists of PMSG (permanent magnet synchronous generator) which is a synchronized steady output at variable input to get constant power output the simulation model of proposed PMSG based wind turbine.

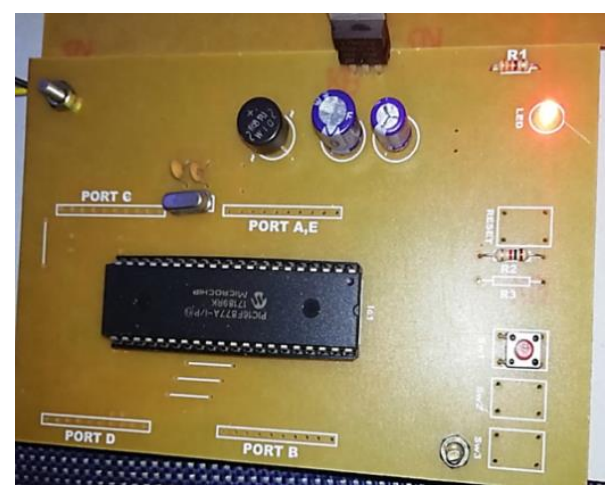

Fig.5 PIC Microcontroller hardwired Model

The power generated by solar and wind power system is coupled to the interleaved boost converter which is controlled using a centralized FLC controller as shown in the figure 5.The Hardware model is the MPPT with FLC implementation for the generation of pulse for boost converter.

The voltage converter DC/DC will boost the voltage for the required level using the pulse from MPPT-FLC combination technique which produces an efficient way of dc output voltage generation. The gate driver circuit diagram is as shown in the figure 6 . 


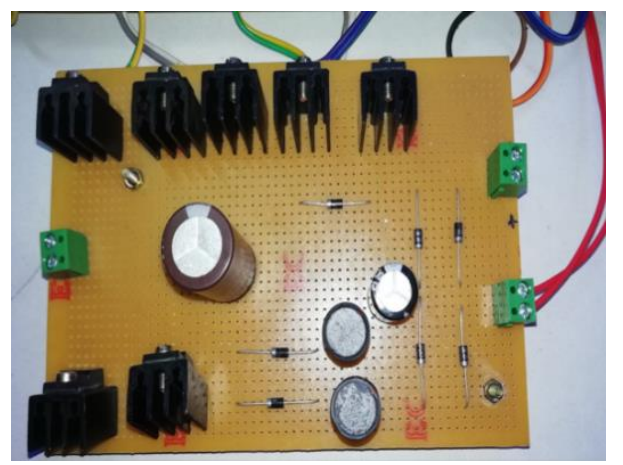

Fig.6 PMSG base wind turbine simulation model

\section{Hardware Model Results}

The Hardware is done through power electronics component it gives good platform for engineering design in mathematical. The project is designed and modeled using power electronics components and the output is made through millimeter and CRO. Input solar DC voltage waveform is as shown in the figure 7.

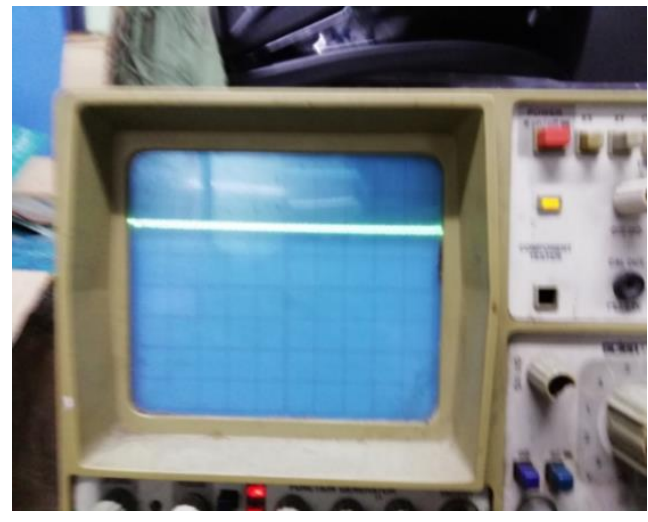

Fig.7. Solar output voltage waveform

The wind power is modeled as single phase AC output voltage waveform is as shown in the figure 8.

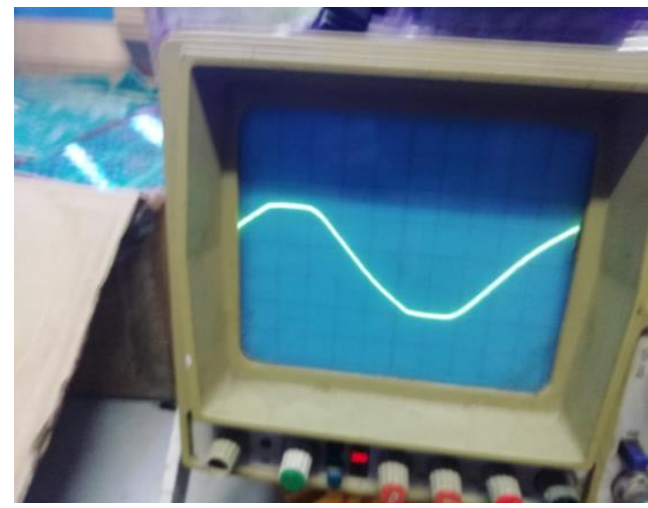

Fig.8 Wind output voltage waveform

The PWM pulse waveform is as shown in the figure 9. 


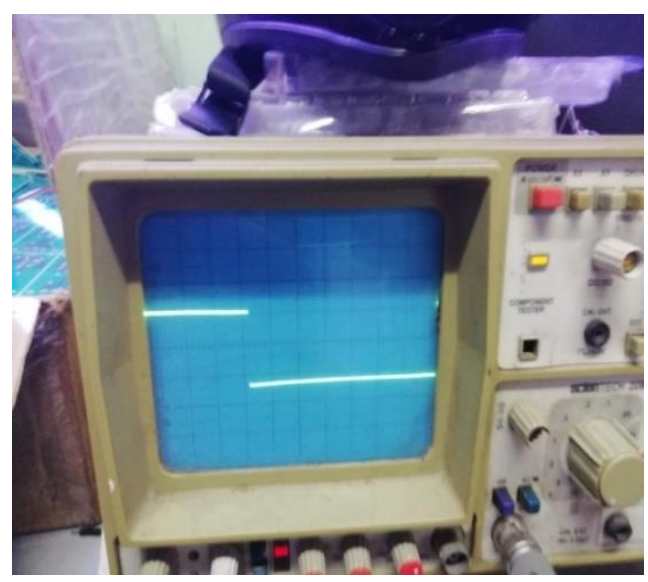

Fig.9. PWM pulse output

\section{Conclusions}

From then on top of simulation results and output graphs we are able to return to a conclusion that out projected methodology of hybrid wind and solar energy generations output voltage is proscribed because of its nature. This condition is overwhelmed with the help of our proposed MPPT controller with FLC implementation in interleaved boost converter. This proposed technique has proved to be the best technique as compared to the traditional way of extracting maximum power from the hybrid wind solar generation system. The voltage gathered from wind and solar to be less as of 12 volt which is increased to about 113 volt as shown in the simulation diagram. This proposed method provides less ripple and harmonics which is also verified using the simulated proposed system. The scope of this project can also expanded to very lengthy extent as the application of renewable energy poses good innovative technology in upcoming days.

\section{References}

[1] S. Prakash \& S.P.Vijayaragavan, "Design and Optimization of High Efficient Charge Controller for a Solar Photovoltaic System Power Generation", International Journal of Pure and Applied Mathematics, Volume 119 No. 12 2018, 4057-4065.

[2] S. Prakash \& V.Jayalakshmi, "Hybrid Solar-Wind Energy System with Mppt Using Cuk- Sepic Fused Converter", International Journal of Pure and Applied Mathematics, Volume 119 No. 12 2018, 6851-6859.

[3] S. Prakash \& K.Sakthivel, “APWM Based Multiple Output ZVS DC/DC Converter”, International Journal of Pure and Applied Mathematics, Volume 119 No. 12 2018, 7665-7671.

[4] S. Prakash \& K.Sakthivel, "Battery Energy Storage System for A Stand Alone Windmill -Based On State Of-Charge (SOC) Balancing Control", International Journal of Pure and Applied Mathematics, Volume 119 No. 12 2018, 7691-7700.

[5] Balachandra Pattanaik,"Maximum Intermediate Power Tracking For Renewable energy Service" to publish in published in Springer Lecture Notes on Data Engineering and Communications Technologies (ISSN: 2367-4512),2020,pp462-468

[6] Balachandra Pattanaik, etal. ,"Zeta DC-DC Converter Based on MPPT Technique for BLDC Application"MC Square Scientfic Resarch Vol. 11,No.2,2019pp. 1-12

[7] Balachandra Pattanaik, etal. "Fault Detection and Recovery in Automotive Embedded System using Rough and Fuzzy Rough Sets" LNNS SPRINGER PUBLICATION, SINGAPORE Pte Ltd. 2018Volume - 1, pp. 233-242.

[8] Balachandra Pattanaik, etal. "SEPIC converter with PV system BLDC motor control for bidirectional power grid using sliding mode controller", www.jcreview.com/index.php?mno ISSN2394-5125 VOL 7, ISSUE 11, 2020, pp. 3962-3967 
[9] Balachandra Pattanaik, etal. International Conference on "Fault Detection in Embedded System Using Rough and Fuzzy Rough Sets" WSEAS-2011,ACM Digital library (paper reference number 659-549),pp. 405-411. 